\title{
Seeing is believing: new methods for in situ single-cell transcriptomics
}

\author{
Gal Avital, Tamar Hashimshony and Itai Yanai*
}

\begin{abstract}
New methods employ RNA-seq to study single cells within complex tissues by in situ sequencing or mRNA capture from single photoactivated cells.
\end{abstract}

\section{Introduction}

An old adage relates how a drunk looks for his car keys under a lamppost in the middle of the night - when asked why he searches for the keys many meters away from his car, where they presumably lie, the drunk answers that it is 'too dark over there'! The transcriptome defined as the set of all transcripts in a given sample - can be cartooned as that lamppost. Unlike the proteome or the metabolome, the transcriptome has the unique property of being quantifiable thanks to the relative ease of working with nucleic acids. Microarrays first took advantage of strand complementarity in the 1990s for global gene-expression measurements, and, more recently, with drops in the cost of sequencing, RNA sequencing (RNA-seq) is now commonly used to profile the transcriptome with unmatched resolution. Two new singlecell transcriptomics methods further advance RNA-seq by empowering the study of transcripts in their native environments [1,2].

\section{The complexities of transcriptomics}

As bright as the light cast by the transcriptome appears, it must be acknowledged that it does not always translate to biological function. Nonetheless, the transcriptome is attractive because it is arguably the first phenotype of the genome and it comprises the many recently recognized groups of RNA, such as long non-coding RNAs and microRNAs, in addition to protein-coding RNAs.

Examining transcriptomes in a complex, multicellular sample often involves painful compromises. For example,

\footnotetext{
* Correspondence: yanai@technion.ac.il

Department of Biology, Technion - Israel Institute of Technology, Haifa
} 32000 , Israel

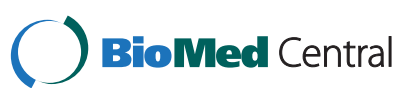

when analyzing the expression profile of a gene in an embryonic time-course, not knowing which specific cell types within the embryo are expressing the gene is a severe handicap. Harnessing methods that can monitor gene expression at the level of individual cells thus holds great promise for resolving the details of cell-type-specific expression. Moreover, even in a population of a given cell type, variation in gene expression can illuminate important functional characteristics. For example, Shalek et al. recently examined 18 cells and found extensive evidence for bimodal gene expression within a population of immune cells [3]. Characterizing such heterogeneity at a global level could further expose unsuspected regulatory mechanisms.

An amplification step exploiting the strand complementarity of nucleic acids is necessary in all single-cell transcriptome methods (Figure 1a-c), including those discussed below. In this context, James Eberwine first introduced transcriptome amplification using $\mathrm{T} 7$ in vitro transcription (IVT) [4]. For a single cell, three rounds of IVT are required, yet the advantage of this approach remains, in that amplification is linear, as opposed to the exponential phase of PCR. IVT was used widely in most gene-expression microarray platforms. With the introduction of RNA-seq, samples can be molecularly barcoded, as in a recently introduced CEL-Seq method, allowing for many single cells to be processed in parallel and thereby requiring only a single round of IVT [5]. PCR-based single-cell transcriptome methods are also available, each with distinct advantages [6-8]. More recently, the notion of unique molecular identifiers (known as UMIs) was introduced to tag transcripts before the reverse-transcription step, allowing for measurement of individual transcripts with dramatically reduced amplification bias [9].

\section{Single-cell transcriptomics in a complex tissue}

In many projects, a particular cell type in a population constitutes a rare subset. Examining these cells individually might mean excising them from their local tissue 
(a) Previous methods
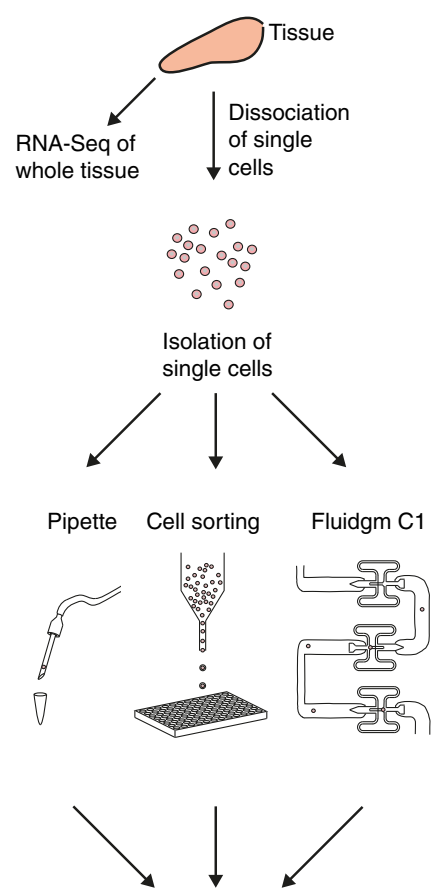

RT

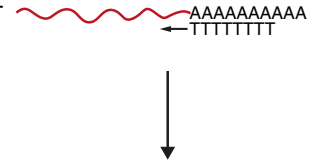

Amplification of RNA by PCR or IVT, library preparation and sequencing (b)

TIVA

TIVA

TIVA tag

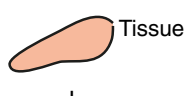

$+$

biotin
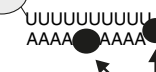

photocleavable linkers

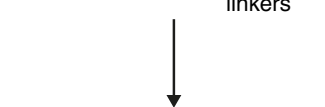

Photoactivation

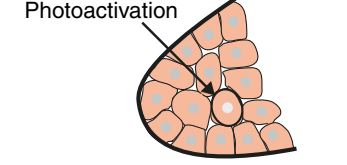
RNA
purification

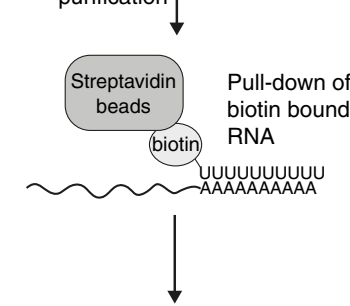

$R T \sim \sim \sim \sim \sim \sim A A A A A A A A$

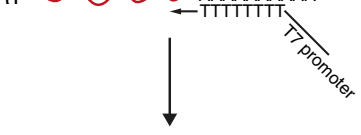

Amplification of RNA by IVT, library preparation and sequencing

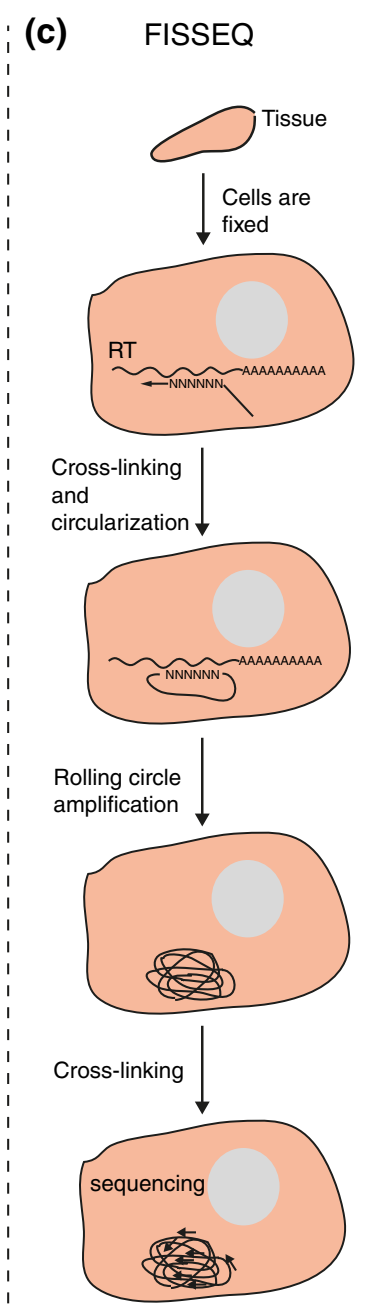

Figure 1 New methods enable single-cell transcriptomics profiling of a complex tissue. (a) In single cell approaches, canonically, individual cells are first dissociated and then isolated by micropipette, automated cell sorting, or microfluidics-enabled capturing. Cellular RNAs are then amplified and sequenced. (b) The transcriptome of an individual cell can be determined by first photoactivating a TIVA-tag within that cell, allowing it to hybridize to cellular mRNAs. These mRNAs can then be specifically purified and sequenced by any RNA-seq method. (c) FISSEQ allows for RNA-Seq within the context of the cell by first fixing mRNA and then amplifying (by rolling circle amplification) and sequencing by the SOLiD technology. FISSEQ: fluorescent in situ RNA sequencing; IVT: in vitro transcription; PCR, polymerase chain reaction; RNA-seq: RNA sequencing; RT: reverse transcription; SOLiD; sequencing by oligonucleotide ligation and detection; TIVA: transcriptome in vivo analysis.

environment and thus compromising the quality of their transcriptomes. A recently published method from Lovatt et al. now allows examination of the transcriptome of an individual cell in a complex tissue by simply shining a laser upon it [1]. This is made possible by washing a live tissue with a transcriptome in vivo analysis (TIVA) tag, a cleverly designed molecule that, like a Swiss Army knife, possesses many properties: a cellpenetrating peptide, a photocleavable linker, the fluorophores $\mathrm{Cy} 3$ and $\mathrm{Cy} 5$, a poly $(\mathrm{U})$ oligonucleotide and biotin (Figure 1b). Thanks to its cell-penetrating peptide, the TIVA tag can permeate into the cells of a tissue; however, by design, this entry causes the peptide to dissociate from the TIVA tag, trapping it in the cell.
Although all the cells in the tissue will contain TIVA tags, these tags will not hybridize to cellular mRNAs as their poly $(\mathrm{U})$ oligonucleotides, which bind to mRNA poly(A) tails, are normally hidden. Nevertheless, laser photoactivation on a particular cell causes its TIVA tags to come undone, as can be validated by fluorescence resonance energy transfer through exploiting the $\mathrm{Cy} 3$ and Cy5 molecules on the tag. Once exposed, these tags can then anneal to mRNAs in the light-selected cell and, after harvesting RNA from the entire tissue, the desired mRNA can be pulled out by using streptavidin beads and then be sequenced (Figure 1b).

Lovatt et al. applied TIVA to study gene expression in brain slices from mouse and human, where they detected 
expression consistent with neuronal cell types [1]. Most interestingly, significantly more bimodal expression was recorded in cells isolated from live tissues in comparison with single neurons in culture, providing additional examples of the bimodality first characterized by Shalek et al. [3]. An especially useful aspect of the TIVA method is that it can be used to connect the particular morphology of a cell to its transcriptome. The TIVA method is thus the first approach to allow for the study of the transcriptome in cells within intact tissues. This provides a significant advance over methods such as laser capture or isolation by pipette (Figure 1a), which can either injure the cell in the collection process or only partially collect the cell - relevant especially for cells, such as neurons, that possess complex morphologies.

\section{In situ sequencing for all transcripts in individual cells}

As mentioned above, single-cell transcriptomics requires amplification, a step that, until now, has involved RNA collection and thus loss of the cellular context of each RNA. In cases where cellular localization information is desired (of mRNA or protein), in situ hybridization is an option, but the capacity of this approach is limited to only a handful of genes at a time. Recently, Ke et al. introduced a method for sequencing RNA in situ and showed that short 4-bp reads can detect point mutations in selected genes [10]. Meanwhile, Lee et al. have now demonstrated a novel approach that takes this a dramatic step forward by profiling the transcriptome in situ in fixed cells, in a method termed fluorescent in situ RNA sequencing (FISSEQ) [2]. This science-fiction-like method relies on performing RNA-seq directly in the cells of interest, through a combination of in situ RNA amplification in a fixed sample and sequencing by oligonucleotide ligation and detection (SOLiD).

FISSEQ involves the reverse transcription of RNAs in fixed cells with dNTPs enriched with aminoallyl-modified dUTPs (aaUTPs; see Figure 1c). These cDNAs are then fixed to the cell protein matrix using a crosslinker that exploits the NH group of the aaUTPs. Rolling circle amplification on the fixed cDNA produces a long single-stranded cDNA for each transcript that can then be sequenced in situ using the SOLiD sequencing platform.

Lee et al. show that the amplification step is able to work in mouse embryos, mouse brain, Drosophila embryos, HeLa cells, human primary fibroblasts and human induced pluripotent stem cells. For fibroblast cells, the authors also sequence amplicons and report a transcriptome visualized within the confines of cellular membranes. The SOLiD sequencing of these cells yields 27-bp reads for 15,000 amplicons covering 4,000 genes. As the reverse-transcription step begins with random hexamers (as opposed to the poly $(\mathrm{U})$ oligonucleotides often used in other protocols, including TIVA), the transcriptome is expected to include a sizeable fraction of non-mRNA transcripts. Yet, for fibroblasts, Lee et al. reported that $44 \%$ of transcripts were mRNAs, which could be attributed to the enrichment of distinctly localized mRNAs that are more resolvable by FISSEQ. When the transcriptome was again probed during simulated wound healing, the mRNA fraction dropped to $7 \%$. Comparing these transcriptomes, the authors found that the differentially expressed genes matched those previously implicated in wound healing.

A comparison of FISSEQ with traditional RNA-seq showed that the two methods were generally well correlated. However, the correlation was notably poor for RNAs involved in RNA and protein processing, leading the authors to make the interesting suggestion that these RNAs localize to cellular structures that are effectively inaccessible to FISSEQ. The ability to establish the localization of gene expression in many cells in parallel will likely make FISSEQ the method of choice for many single-cell transcriptomics applications.

\section{Discussion}

The holy grail of single-cell transcriptomics is to identify the transcript abundance of all genes in a cell positioned in its native environment across both space and time, and to do so without compromising the viability of the cell. Along these lines, TIVA constitutes a major step forward and should prove invaluable in identifying intercellular relationships by enabling analyses of the transcriptomes of neighboring cells at a high level of resolution. Also among the strengths of TIVA as a technology is its accessibility as it appears reasonably friendly to the uninitiated, relying upon commonly available tools.

A noteworthy strength of the FISSEQ method, meanwhile, is that it promises to reveal the spatial substructure of the transcriptome. Knowledge of where particular transcripts reside in cells could also enable a guilt-byassociation approach to identifying functional relationships between genes. With FISSEQ, one exciting prospect is the high-resolution determination of the characteristic path of a transcript-type in the cell throughout its program. Imagining FISSEQ analysis on thousands of cells, the portfolio of locations of a particular transcript type may be interpreted to reveal its cellular program. Such a brightly lit transcriptome could perhaps unlock some of the remaining mysteries of the cell.

\section{Abbreviations}

aaUTPs: Aminoallyl dUTPs; bp: Base pair; dNTP: Deoxyribonucleotide triphosphate; dUTP: Deoxyuridine triphosphate; FISSEQ: Fluorescent in situ RNA sequencing; IVT: In vitro transcription; PCR: Polymerase chain reaction; RNA-seq: RNA sequencing; SOLiD: Sequencing by oligonucleotide ligation and detection; TIVA: Transcriptome in vivo analysis. 


\section{Competing interests}

The authors declare that they have no competing interests.

Published: 31 March 2014

\section{References}

1. Lovatt D, Ruble BK, Lee J, Dueck H, Kim TK, Fisher S, Francis C, Spaethling JM, Wolf JA, Grady MS, Ulyanova AV, Yeldell SB, Griepenburg JC, Buckley PT, Kim J, Sul JY, Dmochowski IJ, Eberwine J: Transcriptome in vivo analysis (TIVA) of spatially defined single cells in live tissue. Nat Methods 2014, 11:190-196.

2. Lee JH, Daugharthy ER, Scheiman J, Kalhor R, Yang JL, Ferrante TC, Terry R, Jeanty SS, Li C, Amamoto R, Peters DT, Turczyk BM, Marblestone AH, Inverso SA, Bernard A, Mali P, Rios X, Aach J, Church GM: Highly multiplexed subcellular RNA sequencing in situ. Science 2014. doi:10.1126/ science.1250212.

3. Shalek AK, Satija R, Adiconis X, Gertner RS, Gaublomme JT, Raychowdhury R, Schwartz S, Yosef N, Malboeuf C, Lu D, Trombetta JJ, Gennert D, Gnirke A, Goren A, Hacohen N, Levin JZ, Park H, Regev A: Single-cell transcriptomics reveals bimodality in expression and splicing in immune cells. Nature 2013, 498:236-240.

4. Eberwine J, Yeh H, Miyashiro K, Cao Y, Nair S, Finnell R, Zettel M, Coleman P: Analysis of gene expression in single live neurons. Proc Natl Acad Sci U S A 1992, 89:3010-3014.

5. Hashimshony T, Wagner F, Sher N, Yanai I: CEL-Seq: single-cell RNA-Seq by multiplexed linear amplification. Cell Rep 2012, 2:666-673.

6. Goetz JJ, Trimarchi JM: Transcriptome sequencing of single cells with Smart-Seq. Nat Biotechnol 2012, 30:763-765.

7. Tang F, Lao K, Surani MA: Development and applications of single-cell transcriptome analysis. Nat Methods 2011, 8:S6-S11.

8. Islam S, Kjällquist U, Moliner A, Zajac P, Fan JB, Lönnerberg P, Linnarsson S: Characterization of the single-cell transcriptional landscape by highly multiplex RNA-seq. Genome Res 2011, 21:1160-1167.

9. Kivioja T, Vähärautio A, Karlsson K, Bonke M, Enge M, Linnarsson S, Taipale J: Counting absolute numbers of molecules using unique molecular identifiers. Nat Methods 2012, 9:72-74.

10. Ke R, Mignardi M, Pacureanu A, Svedlund J, Botling J, Wählby C, Nilsson M: In situ sequencing for RNA analysis in preserved tissue and cells. Nat Methods 2013, 10:857-860.

doi:10.1186/gb4169

Cite this article as: Avital et al.: Seeing is believing: new methods for in situ single-cell transcriptomics. Genome Biology 2014 15:110. 